Vietnam Journal of Mechanics, VAST, Vol.31, No. 1 (2009), pp. $17-30$

\title{
NON-LINEAR BUCKLING ANALYSIS OF FUNCTIONALLY GRADED SHALLOW SPHERICAL SHELLS
}

\author{
Dao Huy Bich \\ Vietnam National University, Hanoi
}

\begin{abstract}
In the present paper the non-linear buckling analysis of functionally graded spherical shells subjected to external pressure is investigated. The material properties are graded in the thickness direction according to the power-law distribution in terms of volume fractions of the constituents of the material. In the formulation of governing equations geometric non-linearity in all strain-displacement relations of the shell is considered. Using Bubnov-Galerkin's method to solve the problem an approximated analytical expression of non-linear buckling loads of functionally graded spherical shells is obtained, that allows easily to investigate stability behaviors of the shell.
\end{abstract}

\section{INTRODUCTION}

A new class of materials, called functionally graded materials (FGMs), has received considerable attention for improved structural efficiency in space structures and nuclear reactors. FGMs are microscopically inhomogeneous, in which the material properties vary smoothly and continuously from one surface of the material to the other surface. This is achieved by gradually varying the volume fractions of the constituent materials. These materials are made from a mixture of ceramic and metal, or combination of different materials [1].

In recent years, important studies have been researched about the stability and vibration of functionally graded plates and cylindrical shells. Birman [2] presented a formulation of the slitability problem for functionally graded hybrid composite plates subjected to uniaxial compression. Feldman and Aboudi [3] studied elastic bifurcation of functionally graded plates acted on by compressive loading. Reddy et al [4] gave bending solution for FGM circular plates and annular plates. In the works [5, 6] a free vibration analysis of FGM cylindrical thin shells under various boundary conditions was investigated.. Woo and Meguid [7] presented an analytical solution for non-linear analysis of FGM plates and shallow cylindrical shells. Yang and Shen [8], Shen [9] gave a large deflection and postbuckling analysis of FGM plates and cylindrical shells subjected to various loadings. Sofiyev [10] studied stability of FGM cylindrical shells under torsional loading and Naj et al [11] investigated conical shells under external pressure. However the non-linear buckling analysis of FGM spherical shells has received comparatively a little attention, this may be because of geometry complexity. Stability problems were investigated for isotropic elastic spherical shells in $[12,13,14]$, for orthotropic elastic shells in [15] and for elasts-plastic 
spherical shells in [16]. Ganapathi et al [17] and Ganapahi [18] presented non-linear buckling analysis of laminated composite and FGM shallow spherical shells by using finite element method with three-noded shear flexible axisymmetric curved shell element, but geometric non-linearity is assumed in strain-displacement relations only on the meridional direction.

In the present paper the non-linear buckling analysis of functionally graded spherical shells is developed based on the approximated analytical method. Geometric non-linearity is assumed in all strain displacement relations. The material properties are graded in the thickness direction according to power-law distribution in terms of volume fractions of the constituents of the material. The non-linear governing equations derived are solved employing Bubnov-Galerkin's method. An approximated analytical expression of buckling critical loads obtained can be used for analyzing stability behaviors of functionally graded shallow spherical shells.

\section{GOVERNING EQUATIONS}

Consider an axisymmetric functionally graded shallow spherical shell of thickness $h$, base radius $r_{0}$, shell radius $R$, made of a mixture of ceramics and metals with the coordinates $\varphi, \theta$ and $z$ along the meridional, circumferential and radial-thickness directions, respectively as shown in Fig. 1

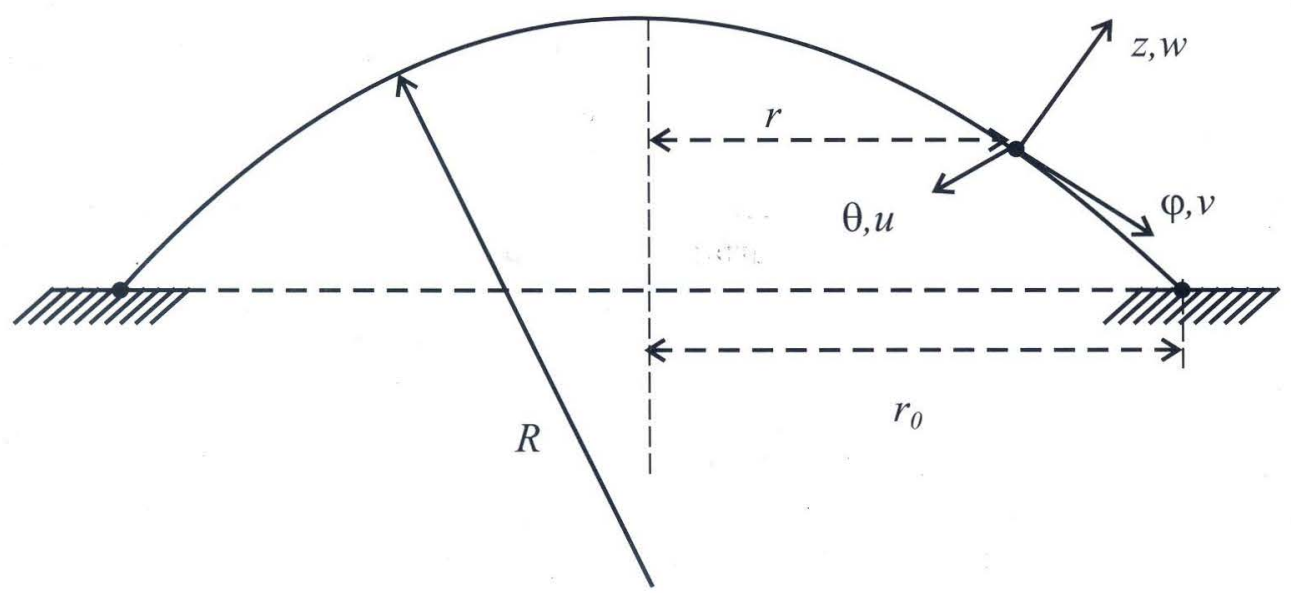

Fig. 1. Geometry and the coordinate system of a spherical cap

The materials in outer $\left(y=\frac{h}{2}\right)$ and inner $\left(y=-\frac{h}{2}\right)$ surfaces of the spherical shells are ceramic and metal respectively. The ceramic constituent of the material provides the hightemperature resistance due to its low thermal conductivity. The ductile metal constituent, on the other hand, prevents fracture caused by stress due to high-temperature gradient in a very short period of time. A such mixture of ceramic and metal with a continuously varying volume fraction can be easily manufactured. According to Javaheri and Eslami [19] assume that, the modulus of elasticity $E$ changes in the thickness direction $z$, while Poisson's ratio $\nu$ is assumed to be constant. 
Denote, that $V_{m}$ and $V_{c}$ are volume-fractions of the metal and ceramic phases respectively. They are related by $V_{c}+V_{m}=1$ and $V_{c}$ is expressed as

$$
V_{c}(z)=\left(\frac{2 z+h}{2 h}\right)^{k}
$$

where $k$ is the volume-fraction exponent $(k \geq 0)$. Then the Young's modulus and Poisson's ratio can be evaluated as following

$$
\begin{aligned}
& E(z)=E_{m} V_{m}+E_{c} V_{c}=E_{m}+\left(E_{c}-E_{m}\right)\left(\frac{2 z+h}{2 h}\right)^{k}, \\
& \nu(z)=\nu .
\end{aligned}
$$

For a shallow spherical shell it is convenient to introduce an additional variable $r$ defined by the relation $r=R \sin \varphi$, where $r$ is the radius of the parallel circle. If the rise of the shell is much smaller than the base radius $r_{0}$ one can take $\cos \varphi \approx 1$ and $R d \varphi=d r$, such that points of the middle surface may be referred to coordinates $r$ and $\theta$. According to the Kirchoff-Love's theory non-linear strain-displacement relations for a shallow spherical shell are of the form

$$
\varepsilon_{i j}=\varepsilon_{i j}^{0}-z \chi_{i j}
$$

where

$$
\begin{aligned}
& \varepsilon_{r}^{0}=\frac{\partial v}{\partial r}-\frac{w}{R}+\frac{1}{2}\left(\frac{\partial w}{\partial r}\right)^{2}, \\
& \varepsilon_{\theta}^{0}=\frac{1}{r}\left(\frac{\partial u}{\partial \theta}+v\right)-\frac{w}{R}+\frac{1}{2}\left(\frac{1}{r} \frac{\partial w}{\partial \theta}\right)^{2}, \\
& \gamma_{r \theta}^{0}=r \frac{\partial}{\partial r}\left(\frac{u}{r}\right)+\frac{1}{r} \frac{\partial v}{\partial \theta}+\frac{1}{r} \frac{\partial w}{\partial \theta} \frac{\partial w}{\partial r}, \\
& \chi_{r}=\frac{\partial^{2} w}{\partial r^{2}}, \\
& \chi_{\theta}=\frac{1}{r^{2}} \frac{\partial^{2} w}{\partial \theta^{2}}+\frac{1}{r} \frac{\partial w}{\partial r}, \\
& \chi_{r \theta}=\frac{1}{r} \frac{\partial^{2} w}{\partial r \partial \theta}-\frac{1}{r^{2}} \frac{\partial w}{\partial \theta},
\end{aligned}
$$

where $u, v$ and $w$ are displacements of the middle surface points along circumferential, meridional and radial directions, respectively; $\varepsilon_{r}^{0}, \varepsilon_{\theta}^{0}, \gamma_{r \theta}^{0}$ and $\chi_{r}, \chi_{\theta}, \chi_{r \theta}$ denote the strains in the middle surface and the changes of curvatures and twist, respectively. They must be relative in the deformation compatibility equation

$$
\frac{1}{r^{2}} \frac{\partial^{2} \varepsilon_{r}^{0}}{\partial \theta^{2}}-\frac{1}{r} \frac{\partial \varepsilon_{r}^{0}}{\partial r}+\frac{1}{r^{2}} \frac{\partial}{\partial r}\left(r^{2} \frac{\partial \varepsilon_{\theta}^{0}}{\partial r}\right)-\frac{1}{r^{2}} \frac{\partial^{2}}{\partial r \partial \theta}\left(r \gamma_{r \theta}^{0}\right)=-\frac{\Delta w}{R}+\chi_{r \theta}^{2}-\chi_{r} \chi_{\theta}
$$

where $\Delta=\frac{\partial^{2}}{\partial r^{2}}+\frac{1}{r} \frac{\partial}{\partial r}+\frac{1}{r^{2}} \frac{\partial^{2}}{\partial \theta^{2}}$ is a Laplace's operator. 
In [18] geometric non-linearity is assumed only for strain component $\varepsilon_{r}^{0}$, herein for all strain-displacement relations (2), therefore the compatibility equation (4) is more exact.

The constitutive stress-strain equations for the shell material are omitted here for brevity, note however that the Young's modulus $E(z)$ is a power function of $z$. Then integrating the stress-strain equations and their moments through the thickness of the shell $\left(-\frac{h}{2} \leq z \leq \frac{h}{2}\right)$ we obtain the expressions of internal forces and moments resultants

$$
\begin{aligned}
& N_{r}=\frac{E_{1}}{1-\nu^{2}}\left(\varepsilon_{r}^{0}+\nu \varepsilon_{\theta}^{0}\right)-\frac{E_{2}}{1-\nu^{2}}\left(\chi_{r}+\nu \chi_{\theta}\right), \\
& N_{\theta}=\frac{E_{1}}{1-\nu^{2}}\left(\varepsilon_{\theta}^{0}+\nu \varepsilon_{r}^{0}\right)-\frac{E_{2}}{1-\nu^{2}}\left(\chi_{\theta}+\nu \chi_{r}\right), \\
& N_{r \theta}=\frac{E_{1}}{2\left(1+\nu^{2}\right)} \gamma_{r \theta}^{0}-\frac{E_{2}}{1+\nu} \chi_{r \theta},
\end{aligned}
$$

and

$$
\begin{aligned}
& M_{r}=\frac{E_{2}}{1-\nu^{2}}\left(\varepsilon_{r}^{0}+\nu \varepsilon_{\theta}^{0}\right)-\frac{E_{3}}{1-\nu^{2}}\left(\chi_{r}+\nu \chi_{\theta}\right), \\
& M_{\theta}=\frac{E_{2}}{1-\nu^{2}}\left(\varepsilon_{\theta}^{0}+\nu \varepsilon_{r}^{0}\right)-\frac{E_{3}}{1-\nu^{2}}\left(\chi_{\theta}+\nu \chi_{r}\right), \\
& M_{r \theta}=\frac{E_{2}}{2\left(1+\nu^{2}\right)} \gamma_{r \theta}^{0}-\frac{E_{3}}{1+\nu} \chi_{r \theta},
\end{aligned}
$$

where

$$
\begin{aligned}
& E_{1}=\int_{-\frac{h}{2}}^{\frac{h}{2}} E(z) d z=E_{m} h+\frac{\left(E_{c}-E_{m}\right) h}{k+1}=E_{1}^{*} h, \\
& E_{2}=\int_{-\frac{h}{2}}^{\frac{h}{2}} E(z) z d z=\frac{\left(E_{c}-E_{m}\right) k h^{2}}{2(k+1)(k+2)}=E_{2}^{*} h^{2}, \\
& E_{3}=\int_{-\frac{h}{2}}^{\frac{h}{2}} E(z) z^{2} d z=\frac{E_{m} h^{3}}{12}+\left(E_{c}-E_{m}\right) h^{3}\left(\frac{1}{k+3}-\frac{1}{k+2}+\frac{1}{4 k+4}\right)=E_{3}^{*} h^{3},
\end{aligned}
$$

with

$$
\begin{aligned}
& E_{1}^{*}=E_{m}+\frac{E_{c}-E_{m}}{k+1}, \\
& E_{2}^{*}=\frac{\left(E_{c}-E_{m}\right) k}{2(k+1)(k+2)}, \\
& E_{3}^{*}=\frac{E_{m}}{12}+\left(E_{c}-E_{m}\right)\left(\frac{1}{k+3}-\frac{1}{k+2}+\frac{1}{4 k+4}\right) .
\end{aligned}
$$


By use of Eqs. (5) relations (6) can be rewritten in form

$$
\begin{aligned}
& M_{r}=\frac{E_{2}}{E_{1}} N_{r}-\frac{E_{1} E_{3}-E_{2}^{2}}{E_{1}\left(1-\nu^{2}\right)}\left(\chi_{r}+\nu \chi_{\theta}\right), \\
& M_{\theta}=\frac{E_{2}}{E_{1}} N_{\theta}-\frac{E_{1} E_{3}-E_{2}^{2}}{E_{1}\left(1-\nu^{2}\right)}\left(\chi_{\theta}+\nu \chi_{r}\right), \\
& M_{r \theta}=\frac{E_{2}}{E_{1}} N_{r \theta}-\frac{E_{1} E_{3}-E_{2}^{2}}{E_{1}\left(1-\nu^{2}\right)} \chi_{r} .
\end{aligned}
$$

Inversely from Eqs.(5) strains can be expressed through force resultants

$$
\begin{aligned}
& \varepsilon_{r}^{0}=\frac{1}{E_{1}}\left(N_{r}-\nu N_{\theta}\right)+\frac{E_{2}}{E_{1}} \chi_{r}, \\
& \varepsilon_{\theta}^{0}=\frac{1}{E_{1}}\left(N_{\theta}-\nu N_{r}\right)+\frac{E_{2}}{E_{1}} \chi_{\theta}, \\
& \gamma_{r \theta}^{0}=\frac{2(1+\nu)}{E_{1}} N_{r \theta}+\frac{2 E_{2}}{E_{1}} \chi_{r \theta} .
\end{aligned}
$$

The equations of equilibrium of a shallow spherical shell according to Love's theory are following

$$
\begin{aligned}
& \frac{1}{r} \frac{\partial}{\partial r}\left(r N_{r}\right)+\frac{1}{r} \frac{\partial}{\partial \theta}\left(N_{r \theta}\right)-\frac{N_{\theta}}{r}=0 \\
& \frac{1}{r} \frac{\partial}{\partial r}\left(r N_{r \theta}\right)+\frac{1}{r} \frac{\partial N_{\theta}}{\partial \theta}+\frac{N_{r \theta}}{r}=0 \\
& \frac{1}{r}\left[\frac{\partial^{2}}{\partial r^{2}}\left(r M_{r}\right)+2\left(\frac{\partial^{2} M_{r \theta}}{\partial r \partial \theta}+\frac{1}{r} \frac{\partial M_{\theta}}{\partial \theta}\right)+\frac{1}{r} \frac{\partial^{2} M_{\theta}}{\partial \theta^{2}}-\frac{\partial M_{\theta}}{\partial r}\right]+\frac{1}{R}\left(N_{r}+N_{\theta}\right) \\
& +\frac{1}{r} \frac{\partial}{\partial r}\left(r N_{r} \frac{\partial w}{\partial r}+N_{r \theta} \frac{\partial w}{\partial \theta}\right)+\frac{1}{r} \frac{\partial}{\partial \theta}\left(N_{r \theta} \frac{\partial w}{\partial r}+\frac{1}{r} N_{\theta} \frac{\partial w}{\partial \theta}\right)+p=0,
\end{aligned}
$$

where $p$ is an external pressure acted on the shell. By use of Eqs. (10), (11) the equation (12) is rewritten as

$$
\begin{gathered}
\frac{1}{r}\left[\frac{\partial^{2}}{\partial r^{2}}\left(r M_{r}\right)+2\left(\frac{\partial^{2} M_{r \theta}}{\partial r \partial \theta}+\frac{1}{r} \frac{\partial M_{\theta}}{\partial \theta}\right)+\frac{1}{r} \frac{\partial^{2} M_{\theta}}{\partial \theta^{2}}-\frac{\partial M_{\theta}}{\partial r}\right]+\frac{1}{R}\left(N_{r}+N_{\theta}\right) \\
+N_{r} \frac{\partial^{2} w}{\partial r^{2}}+2 \frac{N_{r \theta}}{r} \frac{\partial^{2} w}{\partial r \partial \theta}+\frac{N_{\theta}}{r^{2}} \frac{\partial^{2} w}{\partial \theta^{2}}+\frac{N_{\theta}}{r} \frac{\partial w}{\partial r}-\frac{2}{r^{2}} N_{r \theta} \frac{\partial w}{\partial \theta}+p=0 .
\end{gathered}
$$

Equations (10), (11) are satisfied by introducing the stress function $F$

$$
N_{r}=\frac{1}{r} \frac{\partial F}{\partial r}+\frac{1}{r^{2}} \frac{\partial^{2} F}{\partial \theta^{2}}, \quad N_{\theta}=\frac{\partial^{2} F}{\partial r^{2}}, \quad N_{r \theta}=-\frac{\partial}{\partial r}\left(\frac{1}{r} \frac{\partial F}{\partial \theta}\right) .
$$

The substitution of Eqs. (9) into the compatibility equation (4) and Eqs. (8) into the equation (13), taking into account relations (14) yields a system of equations in terms of 
the stress function $F$ and the deflection $w$

$$
\begin{aligned}
& \frac{1}{E_{1}} \Delta \Delta F=-\frac{\Delta w}{R}+\left(\frac{1}{r} \frac{\partial^{2} w}{\partial r \partial \theta}-\frac{1}{r^{2}} \frac{\partial w}{\partial \theta}\right)^{2}-\frac{\partial^{2} w}{\partial r^{2}}\left(\frac{1}{r^{2}} \frac{\partial^{2} w}{\partial \theta^{2}}+\frac{1}{r} \frac{\partial w}{\partial r}\right) \\
& \frac{E_{1} E_{3}-E_{2}^{2}}{E_{1}\left(1-\nu^{2}\right)} \Delta \Delta w-\frac{1}{R} \Delta F-\left(\frac{1}{r} \frac{\partial F}{\partial r}+\frac{1}{r^{2}} \frac{\partial^{2} F}{\partial \theta^{2}}\right) \frac{\partial^{2} w}{\partial r^{2}}+2\left(\frac{1}{r} \frac{\partial^{2} F}{\partial r \partial \theta}-\frac{1}{r^{2}} \frac{\partial F}{\partial \theta}\right) \frac{\partial^{2} w}{r \partial r \partial \theta} \\
& -\frac{\partial^{2} F}{\partial r^{2}} \frac{\partial^{2} w}{r^{2} \partial \theta^{2}}-\frac{1}{r} \frac{\partial^{2} F}{\partial r^{2}} \frac{\partial w}{\partial r}+\frac{2}{r^{2}}\left(\frac{1}{r^{2}} \frac{\partial F}{\partial \theta}-\frac{1}{r} \frac{\partial^{2} F}{\partial r \partial \theta}\right) \frac{\partial w}{\partial \theta}=p
\end{aligned}
$$

Eqs. (15), (16) combining with boundary conditions allow to analyse buckling state of functionally graded spherical shells.

\section{LINEAR BUCKLING ANALYSIS OF FUNCTIONALLY GRADED SPHERICAL SHELLS}

Consider a functionally graded spherical shell subjected to an external uniform pressure $p$. The pre-buckling state of the shell can be obtained as $N_{r}^{0}=N_{\theta}^{0}=-p \frac{R}{2}, N_{r \theta}^{0}=$ 0 , where $N_{r}^{0}, N_{\theta}^{0}, N_{r \theta}^{0}$ are membrane forces for the condition with zero initial moments respectively.

The linear stability equations may be derived by application of the adjacent equilibrium criterion. In this purpose we put $u=u_{0}+\delta u v=v_{0}+\delta v, w=w_{0}+\delta w$, where $\left(u_{0}, v_{0}, w_{0}\right)$ represent the equilibrium configuration whose stability is under consideration, $(u, v, w)$ is an adjacent equilibrium configuration corresponding to the same value of applied load as configuration $\left(u_{0}, v_{0}, w_{0}\right)$ and $(\delta u, \delta v, \delta w)$ is an arbitrary small incremental displacement. Furthermore $\delta N_{r}, \delta N_{\theta}, \delta N_{r \theta}, \delta M_{r}, \delta M_{\theta}, \delta M_{r \theta}$ are generalized forces and moment increments corresponding to $(\delta u, \delta v, \delta w)$. Generalized forces and moments increments $\delta N_{i}$ and $\delta M_{i}$ have the form such as (8) and (9).

Omitting non-linear terms in governing equations and repeating the procedure as above from equations (13) and (15) the solvable equations for increments in this case can be obtained

$$
\begin{aligned}
& \frac{1}{E_{1}} \Delta \Delta(\delta F)=-\frac{\Delta(\delta w)}{R} \\
& \frac{E_{1} E_{3}-E_{2}^{2}}{E_{1}\left(1-\nu^{2}\right)} \Delta \Delta(\delta w)-\frac{1}{R} \Delta(\delta F)+\frac{p R}{2} \Delta(\delta w)=0 .
\end{aligned}
$$

Suppose the shell is clamped at its edge $r=r_{0}$, boundary conditions are:

$$
\delta w=0, \quad \frac{\partial}{\partial r}(\delta w)=0 \text { and } \delta N_{r}=\frac{1}{r} \frac{\partial}{\partial r}(\delta F)+\frac{1}{r^{2}} \frac{\partial^{2}}{\partial \theta^{2}}(\delta F)=0 \text { at } r=r_{0} .
$$

Taking the solution

$$
\delta w=\sum_{n=1} \xi_{n} W(r) \sin n \theta, \quad \delta F=\sum_{n=1} \eta_{n} f(r) \sin n \theta
$$


and substituting them into equations (17), (18) yields

$$
\begin{aligned}
& \frac{1}{E_{1}} \eta_{n}\left[f^{I V}+\frac{2}{r} f^{\prime \prime \prime}-\frac{1}{r^{2}} f^{\prime \prime}+\frac{1}{r^{3}} f^{\prime}-2 n^{2}\left(\frac{1}{r^{2}} f^{\prime \prime}-\frac{1}{r^{3}} f^{\prime}+\frac{2}{r^{4}} f\right)+\frac{n^{4}}{r^{4}} f\right] \\
& =-\frac{1}{R} \xi_{n}\left(W^{\prime \prime}+\frac{1}{r} W^{\prime}-\frac{n^{2}}{r^{2}} W\right) ; \\
& \frac{E_{1} E_{3}-E_{2}^{2}}{E_{1}\left(1-\nu^{2}\right)} \xi_{n}\left[W^{I V}+\frac{2}{r} W^{\prime \prime \prime}-\frac{1}{r^{2}} W^{\prime \prime}+\frac{1}{r^{3}} W^{\prime}-2 n^{2}\left(\frac{1}{r^{2}} W^{\prime \prime}-\frac{W^{\prime}}{r^{3}}+\frac{2}{r^{4}} W\right)+\frac{n^{4}}{r^{4}} W\right] \\
& +\frac{p R}{2}\left(W^{\prime \prime}+\frac{1}{r} W^{\prime}-\frac{n^{2}}{r^{2}} W\right) \xi_{n}-\frac{1}{R} \eta_{n}\left(f^{\prime \prime}+\frac{1}{r} f^{\prime}-\frac{n^{2}}{r^{2}} f\right)=0 .
\end{aligned}
$$

Boundary conditions (19) are satisfied if chosen

$$
W(r)=r^{2}\left(r_{0}-r\right)^{2}, \quad f(r)=r^{2}\left(r_{0}-r\right)^{2} .
$$

Substituting these expressions into Eqs. (20), (21) and applying Bubnov-Galèrkin's method in the range $0 \leq r \leq r_{0}$ we obtain

$$
\begin{aligned}
& \eta_{n}=\frac{E_{1} r_{0}^{2}}{R} \cdot \frac{2\left(3+2 n^{2}\right)}{7\left(38+5 n^{2}+12 n^{4}\right)} \xi_{n} \\
& \frac{E_{1} E_{3}-E_{2}^{2}}{E_{1}\left(1-\nu^{2}\right)} 7\left(38+5 n^{2}+12 n^{4}\right) \xi_{n}-p R\left(3+2 n^{2}\right) r_{0}^{2} \xi_{n}=-\frac{2\left(3+2 n^{2}\right)}{R} r_{0}^{2} \eta_{n}
\end{aligned}
$$

from that

$$
p=\frac{2}{R^{2}}\left[\frac{E_{1} E_{3}-E_{2}^{2}}{E_{1}\left(1-\nu^{2}\right)} \cdot \frac{7 R\left(38+5 n^{2}+12 n^{4}\right)}{\left(6+4 n^{2}\right) r_{0}^{2}}+E_{1} \frac{\left(6+4 n^{2}\right) r_{0}^{2}}{7 R\left(38+5 n^{2}+12 n^{4}\right)}\right]
$$

Putting

$$
X=\frac{7 R\left(38+5 n^{2}+12 n^{4}\right)}{\left(6+4 n^{2}\right) r_{0}^{2}} \quad \text { leads to } p=\frac{2}{R^{2}}\left(\frac{E_{1} E_{3}-E_{2}^{2}}{E_{1}\left(1-\nu^{2}\right)} X+\frac{E_{1}}{X}\right) .
$$

An approximate expression for the critical pressure may be obtained by minimization of $p$ with respect to $X$. The smallest $p$ is found as following

$$
p_{c r}=\frac{4}{R^{2}}\left(\frac{E_{1} E_{3}-E_{2}^{2}}{1-\nu^{2}}\right)^{1 / 2}=4\left(\frac{h}{R}\right)^{2}\left(\frac{E_{1}^{*} E_{3}^{*}-E_{2}^{* 2}}{1-\nu^{2}}\right)^{1 / 2} .
$$

In particular case, the shell is made of homogeneous elastic material $E_{c}=E_{m}=E$, so that $E_{1}^{*}=E h, E_{2}^{*}=0, E_{3}^{*}=\frac{E h^{3}}{12}$, the equation (23) gives

$$
p_{c r}=\frac{2 E}{\sqrt{3\left(1-\nu^{2}\right)}}\left(\frac{h}{R}\right)^{2} \text {. }
$$

This is the result given in Timoshenko [13].

The expression for the critical pressure in (23) and (24) is the same as that given for a complete spherical shell. As shown in [14] the critical pressure given by equation (24) is in poor agreement with test data. The discrepancy is due to the neglect of non-linearity in the analysis. 


\section{NON-LINEAR BUCKLING ANALYSIS OF FUNCTIONALLY GRADED SPHERICAL SHELLS}

Taking into consideration of geometrical non-linearity the system of governing equations has of the form (15) and (16)

$$
\begin{aligned}
& \frac{1}{E_{1}} \Delta \Delta F=-\frac{\Delta w}{R}+\left(\frac{1}{r} \frac{\partial^{2} w}{\partial r \partial \theta}-\frac{1}{r^{2}} \frac{\partial w}{\partial \theta}\right)^{2}-\frac{\partial^{2} w}{\partial r^{2}}\left(\frac{1}{r^{2}} \frac{\partial^{2} w}{\partial \theta^{2}}+\frac{1}{r} \frac{\partial w}{\partial r}\right) \\
& \frac{E_{1} E_{3}-E_{2}^{2}}{E_{1}\left(1-\nu^{2}\right)} \Delta w-\frac{1}{R} \Delta F-\left(\frac{1}{r} \frac{\partial F}{\partial r}+\frac{1}{r^{2}} \frac{\partial^{2} F}{\partial \theta^{2}}\right) \frac{\partial^{2} w}{\partial r^{2}}+2\left(\frac{1}{r} \frac{\partial^{2} F}{\partial r \partial \theta}-\frac{1}{r^{2}} \frac{\partial F}{\partial \theta}\right) \frac{\partial^{2} w}{r \partial r \partial \theta} \\
& -\frac{\partial^{2} F}{\partial r^{2}} \frac{\partial^{2} w}{r^{2} \partial \theta^{2}}-\frac{1}{r} \frac{\partial^{2} F}{\partial r^{2}} \frac{\partial w}{\partial r}+\frac{2}{r^{2}}\left(\frac{1}{r^{2}} \frac{\partial F}{\partial \theta}-\frac{1}{r} \frac{\partial^{2} F}{\partial r \partial \theta}\right) \frac{\partial w}{\partial \theta}=p
\end{aligned}
$$

with boundary conditions at clamped base of the shell

$$
w=0, \quad \frac{\partial w}{\partial r}=0, \quad N_{r}=\frac{1}{r} \frac{\partial F}{\partial r}+\frac{1}{r^{2}} \frac{\partial^{2} F}{\partial \theta^{2}}=0 \quad \text { at } \quad r=r_{0} .
$$

An approximation is acceptable in the vicinity of the buckling load. The boundary condition can be satisfied if the buckling mode shape is represented by

$$
w=\xi r^{2}\left(r_{0}-r\right)^{2} \sin n \theta, \quad F=\eta r^{2}\left(r_{0}-r\right)^{2} \sin n \theta .
$$

Applying Bubnov-Galerkin's method to Eqs. (25) and (26) in the range $0 \leq \theta \leq \pi$, $0 \leq r \leq r_{0}$ yields the set of two non-linear algebraic equations with respect to $\xi$ and $\eta$

$$
\begin{aligned}
& \eta=\frac{E_{1}}{38+5 n^{2}+12 n^{4}}\left[\frac{\xi r_{0}^{2}}{7 R}\left(6+4 n^{2}\right)+\frac{\xi^{2} r_{0}^{4}}{63 \pi}\left(284 n-\frac{1600}{n}\right)\right] \\
& \frac{E_{1} E_{3}-E_{2}^{2}}{E_{1}\left(1-\nu^{2}\right)}\left(38+5 n^{2}+12 n^{4}\right) \xi+\frac{\left(6+4 n^{2}\right) r_{0}^{2}}{7 R} \eta-\frac{2 \xi \eta r_{0}^{4}}{63 \pi}\left(\frac{1600}{n}-284 n\right)=\frac{8 p}{\pi n} .
\end{aligned}
$$

Substitution of expression $\eta$ in Eq. (27) into Eq. (28) leads to a non-linear algebraic equation for the pressure $p$

$$
p=\frac{\pi n}{8\left(38+5 n^{2}+12 n^{4}\right)}\left(A \xi-B \xi^{2}+C \xi^{3}\right)
$$

where denote

$$
\begin{aligned}
& A=\frac{E_{1} E_{3}-E_{2}^{2}}{E_{1}\left(1-\nu^{2}\right)}\left(38+5 n^{2}+12 n^{4}\right)^{2}+\frac{E_{1}\left(6+4 n^{2}\right)^{2} r_{0}^{4}}{(7 R)^{2}}, \\
& B=\frac{3 E_{1} r_{0}^{6}\left(6+4 n^{2}\right)\left(1600-284 n^{2}\right)}{63 \pi \cdot 7 R \cdot n}, \quad C=\frac{2 E_{1} r_{0}^{8}\left(1600-284 n^{2}\right)^{2}}{(63 \pi)^{2} \cdot n^{2}} .
\end{aligned}
$$

The equation (29) represents the load-deflection curve of a functionally graded shallow spherical shell. The extremum buckling load of the shell can be found from Eq. (29) using the condition $\frac{d p}{d \xi}=0$, i.e.

$$
3 C \xi^{2}-2 B \xi+A=0
$$


that gives the solution

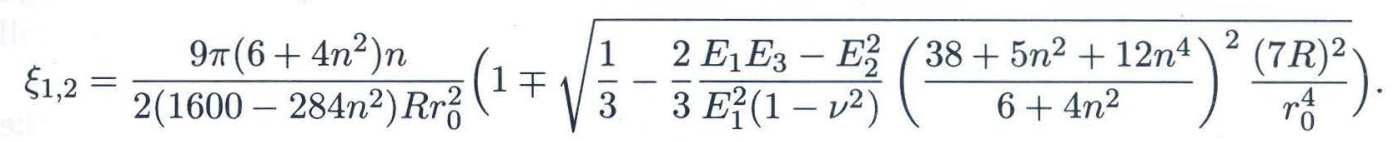

Suppose $r_{0}=\alpha R$, where $\alpha$ is the ratio of the base radius and the shell radius and use of notation (7), Eq. (30) can be rewritten

$$
\xi_{1,2}=\frac{9 \pi\left(6+4 n^{2}\right) n}{2\left(1600-284 n^{2}\right) \alpha^{2} R^{3}}\left(1 \mp \sqrt{\frac{1}{3}-\frac{98}{3} \frac{E_{1}^{*} E_{3}^{*}-E_{2}^{* 2}}{E_{1}^{* 2}\left(1-\nu^{2}\right)}\left(\frac{38+5 n^{2}+12 n^{4}}{6+4 n^{2}}\right)^{2} \frac{1}{\alpha^{4}}\left(\frac{h}{R}\right)^{2}}\right) .
$$

Substitution of (31) into equation (29) yields the buckling critical loads. We can show that $\xi_{1}$ with sign minus before square root corresponds to the upper buckling load $p_{c r}^{(1)}$

$$
\begin{aligned}
& p_{c r}^{(1)}=\frac{9 \pi^{2}\left(6+4 n^{2}\right)^{3} n^{2} E_{1}^{*}}{16\left(1600-284 n^{2}\right)\left(38+5 n^{2}+12 n^{4}\right)} \\
& \times\left[\frac{E_{1}^{*} E_{3}^{*}-E_{2}^{2}}{E_{1}^{* 2}\left(1-\nu^{2}\right)}\left(\frac{38+5 n^{2}+12 n^{4}}{6+4 n^{2}}\right)^{2}\left(\frac{h}{R}\right)^{3} \frac{\Omega_{1}}{\alpha^{2}}+\frac{\alpha^{2}}{98} \frac{h}{R}\left(2 \Omega_{1}-3 \Omega_{1}^{2}+\Omega_{1}^{3}\right)\right],
\end{aligned}
$$

where denote $\Omega_{1}=1-\sqrt{\frac{1}{3}-\frac{98}{3} \frac{E_{1}^{*} E_{3}^{*}-E_{2}^{* 2}}{E_{1}^{* 2}\left(1-\nu^{2}\right)}\left(\frac{38+5 n^{2}+12 n^{4}}{6+4 n^{2}}\right)^{2} \frac{1}{\alpha^{4}}\left(\frac{h}{R}\right)^{2}}$ and $\xi_{2}$ with sign plus before square root corresponds to the lower buckling load $p_{c r}^{(2)}$

$$
\begin{aligned}
& p_{c r}^{(2)}=\frac{9 \pi^{2}\left(6+4 n^{2}\right)^{3} n^{2} E_{1}^{*}}{16\left(1600-284 n^{2}\right)\left(38+5 n^{2}+12 n^{4}\right)} \\
& \times\left[\frac{E_{1}^{*} E_{3}^{*}-E_{2}^{2}}{E_{1}^{* 2}\left(1-\nu^{2}\right)}\left(\frac{38+5 n^{2}+12 n^{4}}{6+4 n^{2}}\right)^{2}\left(\frac{h}{R}\right)^{3} \frac{\Omega_{2}}{\alpha^{2}}+\frac{\alpha^{2}}{98} \frac{h}{R}\left(2 \Omega_{2}-3 \Omega_{2}^{2}+\Omega_{2}^{3}\right)\right],
\end{aligned}
$$

where $\Omega_{2}=1+\sqrt{\frac{1}{3}-\frac{98}{3} \frac{E_{1}^{*} E_{3}^{*}-E_{2}^{* 2}}{E_{1}^{* 2}\left(1-\nu^{2}\right)}\left(\frac{38+5 n^{2}+12 n^{4}}{6+4 n^{2}}\right)^{2} \frac{1}{\alpha^{4}}\left(\frac{h}{R}\right)^{2}}$.

The minimum value of buckling critical loads may be obtained by chosen appropriate value $n$.

The condition provides the existence of upper and lower buckling loads is

$$
98 \frac{E_{1}^{*} E_{3}^{*}-E_{2}^{* 2}}{E_{1}^{* 2}\left(1-\nu^{2}\right)}\left(\frac{38+5 n^{2}+12 n^{4}}{6+4 n^{2}}\right)^{2} \frac{1}{\alpha^{4}}\left(\frac{h}{R}\right)^{2}<1 .
$$

If the inequality (34) is satisfied, the load-deflection curve has following behavior: from the beginning the deflection increases gradually and when it reaches the value $\xi_{1}$, corresponding to the upper buckling load, the active load $p$ becomes decreasing while the deflection continues increasing. It means that at this position the equilibrium shape of the shell becomes unstable and the shell is buckling. Then increasing slightly active load the deflection increases with sudden change to the other value corresponding to the new equilibrium shape. This new shape is stable and increasing active load leads to increasing 
deflection; the sign of the shell curvature has been changed. Here occurs the snap-through phenomenon of the shell. If from this position decreasing active load the deflection will decrease to the value $\xi_{2}$, corresponding to the lower buckling load.

But in this case the active load can not keep the curvature center from above, the lower buckling load is the smallest theoretically possible load corresponding to snap-though of the shell. Consequently, there exists a domain limited by the upper and lower buckling loads, inside which the shell has two equilibrium shapes. This domain is called unstable domain of the shell.

If the shell dimensions and material moduli are such that the expression (34) becomes an equality the load deflection curve has only one stationary point at

$$
\xi_{0}=\frac{9 \pi n\left(3+2 n^{2}\right)}{\left(1600-284 n^{2}\right) \alpha^{2} R^{3}}
$$

and

$$
p_{0}=p\left(\xi_{0}\right)=\frac{9 \pi^{2}\left(3+2 n^{2}\right) n^{2}}{8\left(1600-284 n^{2}\right)} \frac{E_{1}^{*} E_{3}^{*}-E_{2}^{* 2}\left(38+5 n^{2}+12 n^{4}\right)}{E_{1}^{*}\left(1-\nu^{2}\right) \alpha^{2}}\left(\frac{h}{R}\right)^{3} .
$$

It can be seen that this is an inflection point. It means that the load-deflection curve has an inflection point $\xi_{0}$ corresponding to the change of the sign of the shell curvature. This change occurs smoothly without suddenly increasing the deflection when slowly increasing active load. In this case the unstable domain gets narrow to one line and there exists an indifferent equilibrium of the shell.

If the inequality (34) is not satisfied the shell has only one equilibrium shape.

\section{NUMERICAL EXAMPLE}

Consider a ceramic-metal functionally graded shallow spherical shell subjected to uniform external pressure $p$. The combination of materials consists of aluminum and alumina. The Young's modulus of alumina and aluminum are $E_{c}=380 \mathrm{GPa}$ and $E_{m}=70$ GPa, respectively. The Poisson' ratio is chosen to be 0.3 for simplicity. The spherical shell is of uniform thickness and is clamped at its edge. As seen at Eqs. (32) and (33) the critical buckling loads correspond to $n=1$, which is the first mode of buckling. For the shell material we can see that the variation of the composition of ceramics and metal is linear when $k=1$. The value of $k$ equal to zero represents a homogeneous (fully ceramics) shell.

Table 1. Variation of upper critical buckling load versus $R / h$ and $k$

\begin{tabular}{|l|c|c|c|c|c|}
\hline$p_{r}^{(n)}$ & 100 & 200 & 300 & 400 & 500 \\
\hline$p_{r}^{(1)} \times 10^{4}(k=0)$ & 5.1394 & 2.1812 & 1.4091 & 1.0452 & 0.8318 \\
\hline$p_{r}^{(1)} \times 10^{4}(k=1)$ & 2.9419 & 1.2800 & 0.8310 & 0.6174 & 0.4918 \\
\hline$p_{c r}^{(1)} \times 10^{4}(k=2)$ & 2.2717 & 0.9867 & 0.6403 & 0.4757 & 0.3789 \\
\hline
\end{tabular}

Fig. 2 highlights the typical load-deflection curves for the functionally graded spherical shell parameter $\left(\frac{R}{h}=100, r_{0}=\alpha R\right.$ with $\left.\alpha=0.6\right)$ with different power law indices 
Table 2. Variation of lower critical buckling load versus $R / h$ and $k$

\begin{tabular}{|l|c|c|c|c|c|}
\hline$p_{\mathrm{r}}^{(2)} \mathrm{Rh}$ & 100 & 200 & 300 & 400 & 500 \\
\hline$p_{\mathrm{r}}^{(2)} \times 10^{5}(k=0)$ & -6.538 & -16.205 & -12.43 & -9.750 & -7.96 \\
\hline$p_{\mathrm{r}}^{(2)} \times 10^{5}(k=1)$ & -7.061 & -10.005 & -7.481 & -5.825 & -4.739 \\
\hline$p_{\mathrm{r}}^{(2)} \times 10^{5}(k=2)$ & -5.270 & -7.686 & -5.7573 & -4.485 & -3.649 \\
\hline
\end{tabular}

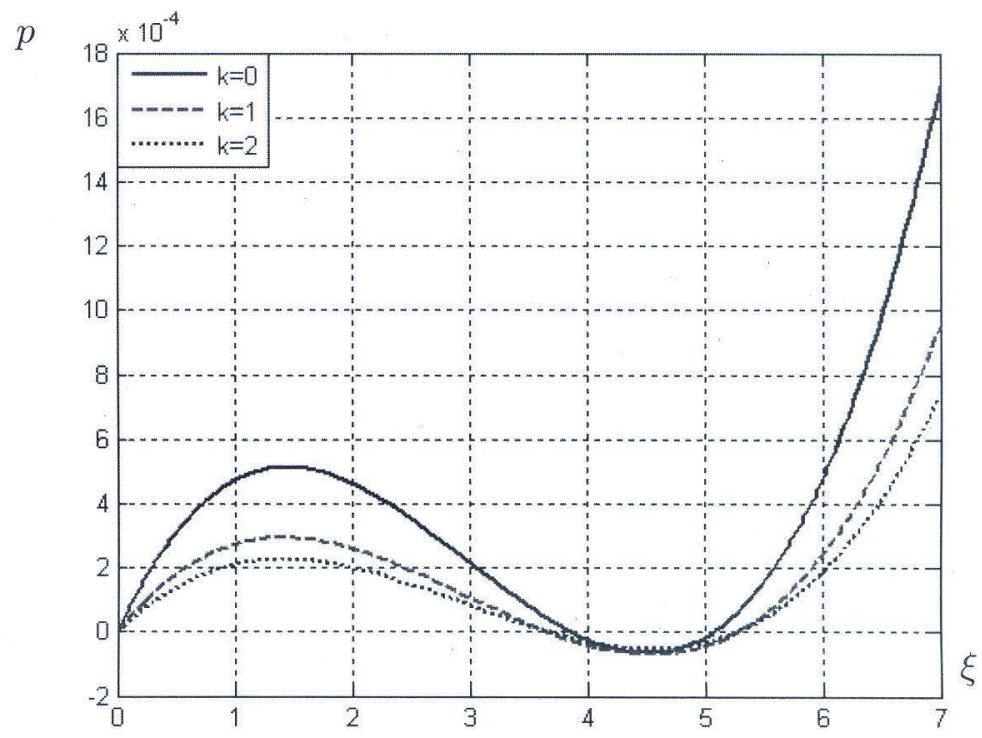

Fig. 2. Load-deflection curves for clamped FGM spherical shell with ratio $R / h=$ $100, \alpha=0.6$ and various $k$

$k$. It shows that the load-deflection curve generally decreases by increasing the power law index $k$.

According to relation (32), (33) calculated values of the upper and lower critical buckling loads of a FGM spherical shell with $r_{0}=0.6 R$, corresponding to different dimension ratios $R / h$ and power law indices $k$, are given in the Tables 1 and 2 and are plotted in Fig. 3 and 4, respectively.

From obtained results one can see that the upper critical buckling load decreases with increasing the dimension ratio $R / h$ and also with increasing the power law index $k$. When $k=0$, representing full ceramic shell, the buckling load is considerably larger than the buckling load of the FGM shells. The reason is the higher value of the assumed modulus of elasticity of the ceramic constituent. What is concerned with lower critical buckling load of FGM spherical shell it requires more detailed investigations. 


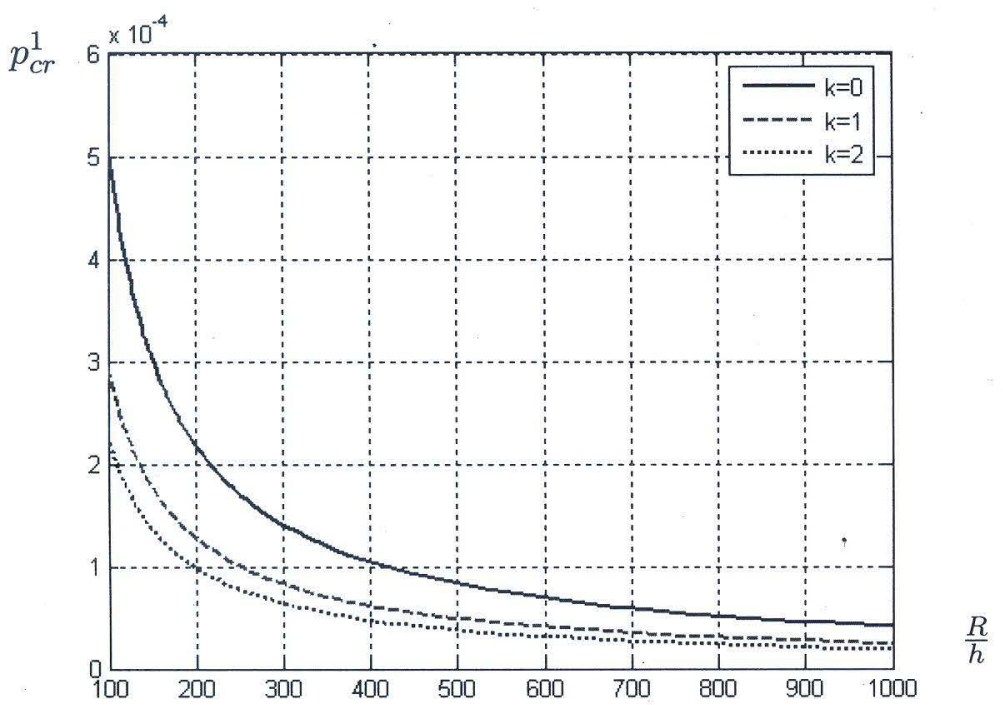

Fig. 3. Upper critical buckling load of FGM spherical shell versus $R / h$ and power law index $k$

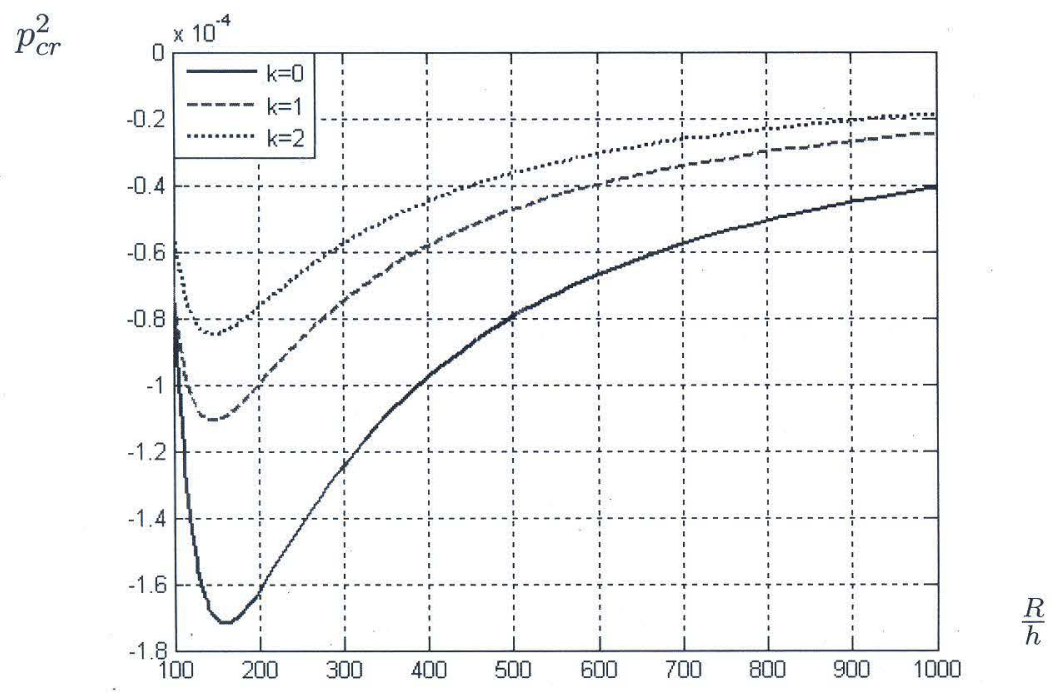

Fig. 4. Lower critical buckling load of FGM spherical shell versus $R / h$ and power law index $k$

\section{CONCLUSIONS}

The governing equations for non-linear buckling analysis of functionally graded spherical shallow shells, including geometric non-linearity, are derived. Derivations are 
based on the classical shell theory and with the assumption of power law composition for the constituent materials. The load-deflection curve of the shell is investigated. The approach to non-linear buckling analysis presented in this paper allows to obtain analytical expressions of critical buckling loads of a clamped FGM spherical shell subjected to external pressure load that provides easily to investigate stability behaviors of the shell. Illustrating numerical results obtained here show that the critical buckling load for the functionally graded spherical shell decreases with increasing dimension ratio $R / h$ and also with increasing the power law index $k$.

\section{ACKNOWLEDGMENT}

The author would like to thank MSc. Nguyen Xuan Nguyen for his help in numerical computations. This paper is completed with partly financial support of VNU project QGTD. 08.07.

\section{REFERENCES}

[1] M. Koizumi, The concept of FGM, Ceram. Trans. Funct Grad. Mater. 34 (1993) 3-10

[2] V. Birman, Buckling of functionally graded hybrid composite plates, Proc. of $10^{\text {th }}$ Conf. on Eng. Mech. Boulder, USA, 1995.

[3] E. Feldman, J. Aboudi, Buckling analysis of FGM plates subjected to uniaxial loading, Composite Structures 38 (1997) 29-36.

[4] J. N. Reddy et al., Axisymmetric bending of FGM circular and annular plates, European J. of Mech. 18 (1999) 185-199.

[5] S. C. Pradham et al., Vibration characteristics of FGM cylindrical shells under various boundary conditions, Applied Acoustics 61 (2000) 119-129.

[6] C. T. Loy et al., Vibration of FGM cylindrical shells, Int. J. Mech. Sci. 1 (1999) 309-324.

[7] J. Woo, S. A. Meguid, Non-linear analysis of FGM plates and shallow shells, Int. J. Solid Struct. 38 (2001) 7409-7421.

[8] J. Yang, H. S. Shen, Non-linear analysis of FGM plates under transverse and in-plane loads, Int. J. Non-linear Mech. 38 (2003) 467-482.

[9] H. S. Shen, Post-buckling analysis of pressure-loaded FGM cylindrical shells in thermal invironments, Eng. Struct. 25 (2003) 487-497.

[10] A. H. Sofiyev, E. Schnack, The stability of FGM cylindrical shells under linearly increasing dynamic torsional loading, Engineering Structures 26 (2004) 1321-1331.

[11] R. Naj, M. Sabzikar, M. R. Eslami, Thermal and mechanical instability of FGM truncated conical shells, Thin-walled Structures 46 (2008) 65-78.

[12] J. R. Fitch, B. Budiansky, Buckling and post-buckling behavior of spherical caps under axisymmetric load, AIAA. Journal 8 (1970) 686-693.

[13] S. P. Timoshenko, J. M. Gere, Theory of elastic stability, Mc. Graw-Hill, 1961.

[14] D. O. Brush, B. O. Almroth, Buckling of bars, plates and shells, Mc. Graw-Hill 1975.

[15] C. C. Chao, I. S. Lin, Static and dynamic snap-through of orthotropic spherical caps, Composite Structures 14 (1990) 281-301.

[16] Dao Huy Bich, On the elasto-platic problem of shells of revolution, Vietnam Journal of Mechanics 25 (2003) 9-18.

[17] M. Ganapathi et al., Non-linear axisymmetric dynamic buckling of laminated angle-ply composite spherical caps, Composite Structures 59 (2003) 89-97. 
[18] M. Ganapathi, Dynamic stability characteristics of functionally graded materials shallow spherical shells, Composite Structures 79 (2007) 338-343.

[19] R. Javaheri, M. R. Eslami, Buckling of FGM plates subjected to uniform temperature rise, Proc. of $4^{\text {th }}$ Int. Congress on thermal stresses, Osaka Japan, June 8-11, 2001, 167-170.

Received June 8, 2009

\section{PHÂN TÍCH PHI TUYẾN VỀ ỔN ĐỊNH CỦA VỎ CẦU THOẢI BẰNG VẠTT LIỆU CÓ CƠ TÍNH BIẾN ĐỔI}

Phân tích phi tuyến ổn định của vỏ cầu thoải bằng vật liệu có cơ tính biến đổi chịu tác dụng của áp suất ngoài được đề cập nghiên cứu trong bài này. Cơ tính của vật liệu biến đổi theo chiều dày của vỏ theo quy luật phân bố phụ thuộc vào thể tích thành phần của các vật liệu tham gia tạo thành vật liệu vỏ. Đã thiết lập các phương trình cơ sở của bài toán khi tính đến phi tuyến hình học trong tất cả các liên hệ giữa biến dạng và chuyển vị. Sử dụng phương pháp Bubnov-Galerkin để giải bài toán đã nhận được biểu thức giải tích gần đúng của lực tới hạn, điều này cho phép dễ dàng khảo sát các tính chất ổn định của, vỏ. 